\title{
Disseminated NK-T Cell Lymphoma Initially Suspected by the Presence of Circulating CD45 bright/CD7 negative NK cells
}

\author{
David Azoulay ${ }^{*}$, Eugene Dementiev ${ }^{2}$, Noa Gross ${ }^{3}$, Revital Saban ${ }^{3}$, Hananya Vaknine ${ }^{4}$, Luiza Akria ${ }^{1}$, Hector I Cohen $^{2}$ and Netanel Horowitz $^{5}$ \\ ${ }^{1}$ Hematology Unit and Laboratories, Galilee Medical Center, Naharia \& Azrieli Faculty of Medicine, Bar-Ilan University, Safed, Israel \\ ${ }^{2}$ Pathology Unit, Galilee Medical Center, Naharia, Israel \\ ${ }^{3}$ Department of Hematology, Hadassah Hebrew University Medical Center, Jerusalem, Israel \\ ${ }^{4}$ Pathology Institute, Wolfson Medical Center, Holon, Israel \\ ${ }^{5}$ Department of Hematology \& BMT, Rambam Health Care Campus, Haifa, Israel
}

\begin{abstract}
$\mathrm{NK} / \mathrm{T}$ cell lymphomas are rapidly progressive related conditions most prevalent among Asian young adults, which may pose a diagnostic challenge due to their unique clinical presentation and rarity. Here we present a case of disseminated NK/T cell lymphoma in a 63-year-old Israeli Sephardic female. The disease initially suspected by flow-cytometry evaluation of CD45 bright/CD7 negative NK cells in the peripheral blood and bone marrow aspiration.
\end{abstract}

\section{Case Report}

A 63-year-old woman was referred to our medical center due to general weakness. Physical examination was remarkable for hepatosplenomegaly and generalized lymphadenopathy. Complete blood cell count demonstrated mild anemia and thrombocytopenia $\left(29 \times 10^{3} /\right.$ $\mu \mathrm{L}$ ). White blood cell count was $5.54 \times 10^{3} / \mu \mathrm{L}$, with a normal absolute lymphocyte count of $1.9 \times 10^{3} / \mu \mathrm{L}$. Peripheral blood (PB) smear showed few atypical large granular lymphocytes. Flow-cytometry of the $\mathrm{PB}$ demonstrated a suspicious cluster of CD 45 bright, sCD3 $/ \mathrm{CD} 7 / \mathrm{CD} 19 \%$ CD56+ cells that accounted for $31 \%$ of total lymphocytes. These cells lacked expression of the NK cells markers CD16 and CD57 (Figure 1A).

A bone marrow (BM) aspiration and biopsy was performed. Flowcytometry of the aspiration demonstrated the same suspicious cluster of CD45 bright sCD3\%/CD7/CD19-CD56+ cells which accounted for $92 \%$ of total lymphocytes. Further analysis confirmed their NK ontogeny as they showed CD2 expression, and lack CD5 expression. Similar to PB, these atypical NK cells showed negative CD16 and CD57 expression. Additional staining showed dim expression of HLA-DR and CD26, and negative expression of TcR $\alpha \beta, \mathrm{TcR} \gamma \delta, \mathrm{CD} 4, \mathrm{CD} 8, \mathrm{CD} 19, \mathrm{CD} 20, \mathrm{CD} 123$ and CD138. Myeloid associated antigens CD33, CD64 and CD14 were negative, although weak CD11b expression observed in $50 \%$ of the cells. Cytoplasmic CD3e was weakly positive whereas $\mathrm{TdT}$ was negative (Figure 1A). PCR analysis of the BM aspiration demonstrated germ line TcR rearrangement.

Histochemical and Immuno-histochemical analysis of the BM biopsy showed hyper-cellularity with diffuse monomorphic infiltrate of blast-like cells, stained positive for LCA, CD2, CD56 and TIA1. CD3 showed focal staining and CD43 was weak in about $50 \%$ of the cells. Granzyme B and Lysozyme were positive in about $50 \%$ of the cells. CD30, c-kit and CD57 were negative and CD4, CD5, CD7, CD8, CD20 and CD79 $\alpha$ staining were observed in a few cells. KI67 was positive in $95 \%$ of total cells. EBV latent membrane protein 1 (LMP-1) was negative. However, EBV detection by in situ hybridization (EBER) was positive in most malignant cells (Figure 2B). PET-CT showed diffuse and intense pathological FDG uptake in the BM, spleen and right inguinal lymph nodes without nasopharyngeal involvement.

A diagnosis of NK/T cell lymphoma was made and the patient was transferred to another hospital. Treatment with the SMILE regimen was considered however due to impaired liver enzymes CHOEP regimen was initiated. Three weeks after initial therapy, CNS involvement was diagnosed by lumbar puncture and cerebrospinal fluid (CSF) examination (Figure 1A). PCR analysis of the CSF for rearrangement show germ line TcR. Therapy with systemic high dose cytarabine and thiothepa was initiated, concomitant with cytarabine administration via Ommaya reservoir placement. Further CSF analysis was negative for malignant cells and neurological symptoms resolved. While initial improvement in CNS involvement, her systemic disease progressed, hence, bendamustin and pembrolizumab were administered, unfortunately with no response. The patient's condition has deteriorated and she succumbs to her disease 4 month after her initial diagnosis.

NK/T cell lymphoma is a distinct entity in the World Health Organization (WHO) classification system characterized by EBV associated lymphoma cells that express cytoplasmic CD3e, CD56 and

${ }^{\star}$ Correspondence to: David Azoulay, Ph.D. Hematology Unit, Galilee Medical Center, P.O.B 21 Naharia, 22100 Israel, Tel: 972-4-910-7657; Fax: 972-4-9107469; E-mail: davidA@GMC.gov.il

Key words: NK-T cell lymphoma, flow cytometry, CD7, CD56, CD57, CD16

Received: March 01, 2021; Accepted: March 29, 2021; Published: March 31, 2021 
A

i
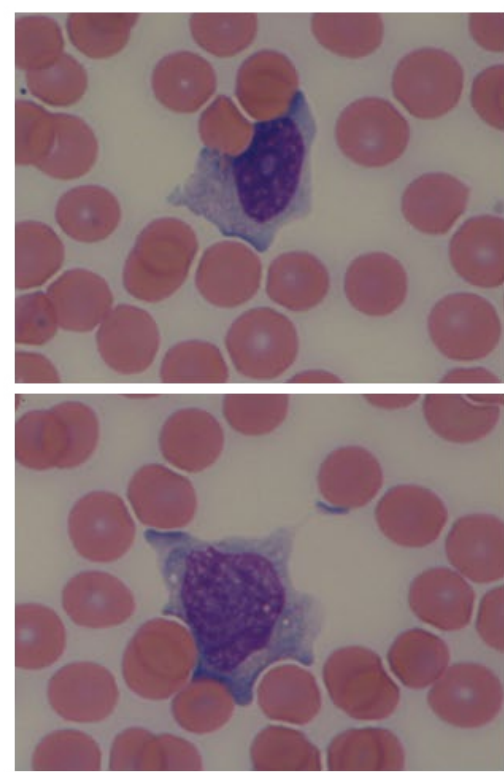

iii
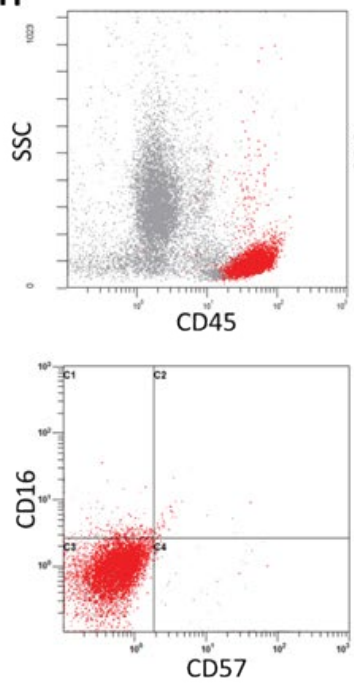

ii
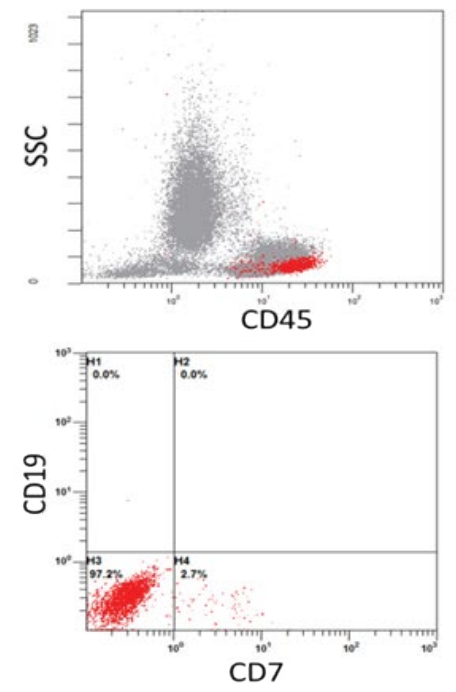

CD7
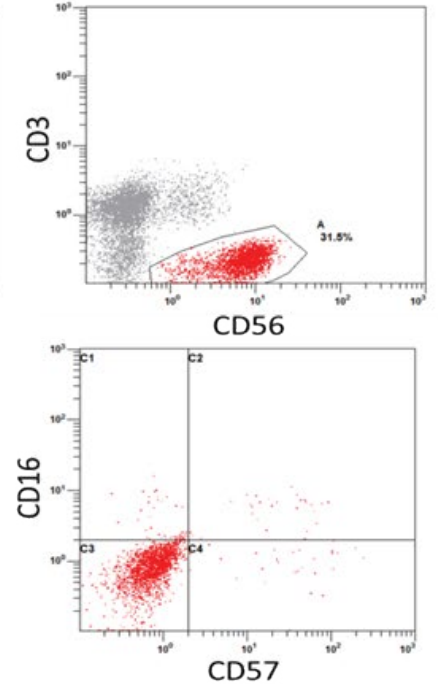

iiii

Figure 1. A. Morphological and immune-phenotypic findings in the PB, BM aspiration and CSF. i PB-smear showing atypical large granular lymphocytes. ii. Flow cytometry of the PB showing the suspected CD45 bright cells on a CD45/SSC profile (upper left plot), with negative surface CD3 expression and positive CD56 expression (upper right plot), with negative surface CD19 and CD7 expression (lower left plot) and negative CD57 and CD16 expression (lower right plot). iii. Flow-cytometry of the BM aspiration showing the suspected CD45 bright cells on a CD45/SSC profile (upper left plot), with negative surface CD3 expression and positive CD56 expression (upper middle plot), with negative surface CD19 and CD7 expression (upper left plot), negative CD57 and CD16 expression (lower left plot), positive CD2 expression and negative CD5 expression (lower middle plot), positive cytoCD3 expression and negative TdT expression (lower right plot). iiii Morphological findings in the CSF showing atypical large granular lymphocytes

cytotoxic molecules. NK/T cell lymphoma may present in three clinical scenarios. Nasal type, involving the upper aero-digestive tract. Nonnasal (extra-nasal) type may involve any site, commonly the skin, gut and testes. The third presentation is the disseminated one characterized by widespread tissue infiltration and marrow involvement with a frequent leukemic phase. Disseminated NK/T cell lymphoma may be indistinguishable from aggressive NK cell leukemia (ANKL); another entity related to NK/T cell lymphoma which is also characterized by widespread tissue infiltration and massive marrow involvement.
Immunophenotypically, NK cells and $\mathrm{T}$ cells share common $\mathrm{T}$ associated antigens including CD2, and CD7. As opposed to T cells, NK cells are negative for $\mathrm{CD} 5$ and surface $\mathrm{CD} 3$, but express cytoplasmic CD3e. CD56 is the most consistent marker expressed in NK cells. As compared to normal, reactive or chronic lymphoproliferative disorder of NK cells (CLPD-NK) increased CD56 is expressed in both NK/T cell lymphoma and ANKL [1]. Reactive or CLPD-NK cells also express CD57 whereas is usually absent in NK/T cell lymphomas. As compared to normal NK cells, NK/T cell lymphomas usually lack both CD16 and 
B
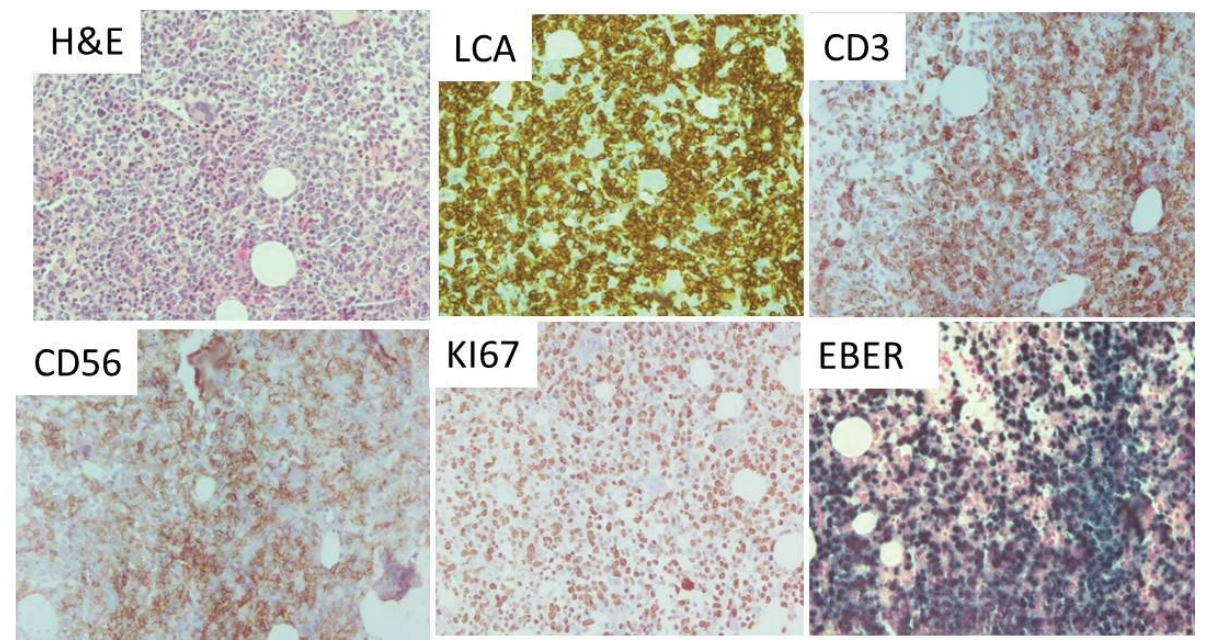

Figure 2. B. Histo-chemical and Immuno-histochemical findings in the BM biopsy. (From left to right) H\&E demonstrating the massive infiltration of the BM with medium size blast-like cells and representative pictures of the immuno-histochemistry for LCA, CD3, CD56, KI67 and EBER -ISH

CD7 [2]. There are some reports suggesting that in ANKL neoplastic cells frequently loss CD7 expression [3]. However, in other series of ANKL CD7 determined to be positively expressed. Of note, it was this bright CD45 expression and the loss of CD7 in the NK cells in our case that was the most important initial observation that raised the suspicion of NK/T cell lymphoma.

Taken into account the massive BM involvement of the NK/T cell lymphoma at presentation coupled with the absence of involvement of sites common in the non-nasal NK/T cell lymphoma favors the diagnosis of disseminated NK/T cell lymphoma in our case. However, due to the very aggressive clinical course of the disease in our patient we cannot rule out the possibility that our case represents ANKL. Although the majority of NK/T cell lymphoma and ANKL cases are from East Asian regions like Japan and China our case diagnosed in Sephardic Israeli patient is akin to other sporadic cases reported in Europe and North America.

Several investigators [3] reported CNS involvement in NK/T cell lymphoma and ANKL. However, despite its clinical importance, little is known about the mechanisms underlying this phenomenon. NK cell activation was shown to be an important mechanism, inhibiting leukemic cells outgrowth. In addition, it was suggested that diminution of NK cells in the CNS compartment may support leukemic cells penetration to this site. Furthermore, depletion of NK cells in NOD/ SCID mice enabled concomitant systemic and CNS involvement of human pre-B ALL, suggesting that intact NK cell activity is essential to prevent CNS penetration by leukemic cells [4]. In our case, replacement of normal NK cell by leukemic counterparts may partially underlined CNS involvement.

Increased expression of interleukin-15 (IL-15) mRNA in ALL blasts was previously reported to be associated with increased risk for CNS involvement. Interestingly it was also shown that IL-15 is up-regulated by therapies that induce lymphoid cell depletion such as cyclophosphamide [5]. Therefore one might suggests that IL-15 up regulation by the lympholytic effect of initial chemotherapy (i.e. CHOEP) could be an additional possible mechanism that facilitated CNS involvement in our case. However, this hypothesis is anecdotal and should be further explored.

\section{Author contribution}

David Azoulay performed flow cytometry analysis, collected the data and wrote the manuscript. Eugene Dementiev and Hector I Cohen performed pathological analysis. Hananya Vaknine helped to interoperate the data and review the manuscript. Noa Gross, Revital Saban and Luiza Akria collected clinical data from the patient. Netanel Horowitz review and wrote the manuscript.

\section{Conflict of interest}

All authors declare no conflict of interest.

\section{References}

1. Jiang NG, Jin YM, Niu Q, Zeng TT, Su J, et al. (2013) Flow cytometric immunophenotyping is of great value to diagnosis of natural killer cell neoplasms involving bone marrow and peripheral blood. Ann hematol 92: 89-96. [Crossref]

2. de Mel S, Li JB, Abid MB, Tang T, Tay HM, et al. (2018) The utility of flow cytometry in differentiating NK/T cell lymphoma from indolent and reactive NK cell proliferations. Cytometry B Clin Cytom 94: 159-618. [Crossref]

3. Yoo EH, Kim HJ, Lee ST, Kim WS, Kim SH (2009) Frequent CD7 antigen loss in aggressive natural killer-cell leukemia: a useful diagnostic marker. Korean J Lab Med 29: 491-496. [Crossref]

4. Frishman-Levy L, Shemesh A, Bar-Sinai A, Ma C, Ni Z, et al. (2015) Central nervous system acute lymphoblastic leukemia: role of natural killer cells. Blood 125: 34203431 .

5. Anthony SM, Rivas SC, Colpitts SL, Howard ME, Stonier SW, et al. (2016) Inflammatory Signals Regulate IL-15 in Response to Lymphodepletion. J Immunol 196: 4544-4552. [Crossref]

Copyright: (C2021 Azoulay D. This is an open-access article distributed under the terms of the Creative Commons Attribution License, which permits unrestricted use, distribution, and reproduction in any medium, provided the original author and source are credited. 